\title{
Politique
}

Politique

\section{Mettre Léviathan aux fers : le libéralisme méthodologique de James Buchanan}

\section{Jean-Guy Prévost}

Numéro 22, automne 1992

URI : https://id.erudit.org/iderudit/040728ar

DOI : https://doi.org/10.7202/040728ar

Aller au sommaire du numéro

Éditeur(s)

Société québécoise de science politique

ISSN

0711-608X (imprimé)

1918-6584 (numérique)

Découvrir la revue

Citer cet article

Prévost, J.-G. (1992). Mettre Léviathan aux fers : le libéralisme méthodologique de James Buchanan. Politique, (22), 25-54. https://doi.org/10.7202/040728ar
Résumé de l'article

Cet article porte sur la pensée politique de l'économiste américain James $\mathrm{M}$. Buchanan, chef de file de l'école du Public Choice et figure de premier plan du courant néo-libéral. Le coeur de cette pensée réside dans sa prétention à déduire la primauté de la liberté individuelle d'une position méthodologique plutôt que d'un raisonnement utilitariste ou d'un a priori métaphysique. D'un côté, en effet, Buchanan insiste fortement sur l'indépendance logique entre l'individualisme entendu comme méthode d'analyse et l'individualisme entendu comme norme régissant les arrangements politiques. De l'autre, il s'efforce d'édifier une sorte de déontologie, dont les traits et les composantes procéderaient de l'individualisme méthodologique, mais dont la validité ne reposerait aucunement sur une préférence pour l'individualisme normatif. Nous entendons démontrer ici que Buchanan n'arrive pas à tenir son pari d'un libéralisme purement méthodologique : la défense du statu quo à laquelle il se livre ne s'explique pas uniquement par le scepticisme moral, mais aussi par la crainte que la mise en oeuvre de changements ne faisant pas consensus ne mine la stabilité du gouvernement et ne conduise à un état d'anarchie encore plus indésirable. 


\title{
METTRE LÉVIATHAN AUX FERS : LE LIBÉRALISME MÉTHODOLOGIQUE DE JAMES BUCHANAN
}

\author{
Jean-Guy Prévost \\ Université du Québec à Montréal
}

\begin{abstract}
Cet article porte sur la pensée politique de l'économiste américain James $M$. Buchanan, chef de file de l'école du Public Choice et figure de premier plan du courant néo-libéral. Le cœur de cette pensée réside dans sa prétention à déduire la primauté de la liberté individuelle d'une position méthodologique plutôt que d'un raisonnement utilitariste ou d'un a priori métaphysique. D'un cóté, en effet, Buchanan insiste fortement sur l'indépendance logique entre l'individualisme entendu comme méthode d'analyse et l'individualisme entendu comme norme régissant les arrangements politiques. De l'autre, il s'efforce d'édifier une sorte de déontologie, dont les traits et les composantes procéderaient de l'individualisme méthodologique, mais dont la validité ne reposerait aucunement sur une préférence pour l'individualisme normatif. Nous entendons démontrer ici que Buchanan n'arrive pas à tenir son pari d'un libéralisme purement méthodologique : la défense du statu quo à laquelle il se livre ne s'explique pas uniquement par le scepticisme moral, mais aussi par la crainte que la mise en œuvre de changements ne faisant pas consensus ne mine la stabilité du gouvernement et ne conduise à un état d'anarchie encore plus indésirable.
\end{abstract}

Peut-on être à la fois libéral et agnostique? Le libéral convaincu n'est-il pas obligé de troquer son scepticisme contre la foi du prosélyte dès qu'on le presse de définir sa conception de la bonne société? James Buchanan, prix Nobel d'économie, chef de file de l'école du Public Choice et figure de proue du néo-libéralisme, répondrait sans doute "oui» à la première question et «non» à la seconde. Depuis toujours, il exhorte les économistes à cesser de proférer des conseils politiques «comme s'ils étaient au service d'un despote bienfaisant" et à s'intéresser plutôt aux mécanismes au moyen desquels se prennent les décisions 
politiques'. Bien qu'il n'hésite pas à exprimer ses inquiétudes sur l'avenir des libertés individuelles dans les sociétés occidentales, il juge «improductif et inintéressant» de chercher à convaincre de la supériorité des valeurs qui lui sont chères ${ }^{2}$. Pour Buchanan, l'économiste politique ne doit pas «jouer à Dieu» : ses préférences ne valent a priori que pour lui seul et, s'il arrive que d'autres les partagent, le renfort qu'elles y gagnent tient à l'accord qui se manifeste et non à une quelconque transcendance intrinsèque. $\mathrm{Ce}$ relativisme axiologique, Buchanan prétend le faire dériver tout entier de l'individualisme méthodologique. Si l'on pose que les phénomènes politiques s'expliquent par les décisions et les actions des individus plutôt que par les soliloques d'une entité supra-individuelle, on doit admettre du même coup que, les individus entretenant des préférences et des valeurs différentes, rien ne permet à l'économiste d'affecter les siennes d'un poids supérieur ${ }^{3}$. L'individu étant l'unique entité dépositaire de conscience et, partant, seul capable de procéder à des évaluations, il n'existe pas d'autres sources de valeurs que les individus eux-mêmes ni de justifications permettant de discriminer entre les préférences et les valeurs exprimées par les divers individus ${ }^{4}$.

L'attitude de Buchanan peut effectivement être décrite comme agnostique en comparaison de celle d'un Hayek (qui présente le libéralisme comme le résultat d'un processus de sélection culturelle), d'un Nozick (dont la préférence pour un État minimal dérive de droits naturels dogmatiquement proclamés mais jamais démontrés), voire d'un Rawls (pour qui les principes de justice ne peuvent surgir que d'un accord entre individus, mais qui

Pour toutes les références qui suivent, le nom de Buchanan ne sera pas mentionné quand celui-ci est l'unique auteur.

1 "The Constitution of Economic Policy", in Buchanan, James M., Economics : Between Moral Philosophy and Predictive Science, College Station, Texas A\&M University Press, 1987, p. 303. Notons qu'il s'agit du discours prononcé par Buchanan lorsqu'il a reçu le prix Nobel d'économie en 1986.

2 The Limits of Liberty. Between Anarchy and Leviathan, Chicago, University of Chicago Press, 1975, p. 1.

3 Ibid.

4 Brennan, Geoffrey et James M. Buchanan, The Reason of Rules. Constitutional Political Economy, Cambridge, Cambridge University Press, 1985, p. 21. 
se substitue à eux - il joue à Dieu, dirait Buchanan - pour fixer lui-même le contenu de ces principes). Mais Buchanan s'en tient-il vraiment à cet agnosticisme déclaré? Un critique attentif n'a pas manqué de relever la profession de foi kantienne qui apparaît dans Freedom in Constitutional Contract («persons are to be treated strictly as ends and never as means") ni l'aveu de Buchanan à l'effet que le concept d'optimalité parétienne, dont il fait grand usage, comporte une dimension éthique ${ }^{5}$. On peut également trouver paradoxale la position d'un auteur qui argue de la neutralité axiologique de ses analyses et appelle du même souffle ses collègues à consacrer plus d'efforts à la formulation de propositions normatives $^{6}$. Mais la clé de voûte de cette philosophie politique réside dans sa prétention à déduire la primauté de la liberté individuelle d'une position méthodologique plutôt que d'un raisonnement utilitariste ou d'un a priori métaphysique. D'un côté, Buchanan insiste fortement sur l'indépendance logique entre l'individualisme conçu comme méthode d'analyse et l'individualisme conçu comme norme régissant les arrangements politiques. De l'autre, il s'efforce d'édifier une sorte de déontologie, dont les traits et les composantes procéderaient de l'individualisme méthodologique, mais dont la validité ne reposerait nullement sur une préférence pour l'individualisme normatif, au point qu'on a pu, dans un article récent, parler à son propos d'un "libéralisme méthodologique» plutôt que proprement politique?. La politique y devient une activité où l'on cherche à s'entendre sur les règles et les moyens, où la discussion sur les fins et la recherche de valeurs transcendantes n'ont pas leur place. On doit reconnaître qu'une telle perspective porte fruit lorsqu'on l'utilise pour fonder des modèles d'analyse positive. L'électeur, le

5 "Democratic Values in Taxation", in Freedom in Constitutional Contract, p. 244. Cité par Barry, Norman, "Unanimity, Agreement and Liberalism: A Critique of James Buchanan's Social Philosophy», Political Theory, 12, 1984, p. 583-594.

- $\quad *(\ldots)$ I think that we must become more normative in our efforts; we should use the results of our positive analysis in the discussion of policy reform. We must use the "is" to implement the "ought" which the "is" suggests, regardless of the methodological impropriety of this relationship (...)". "Notes on the History and Direction of Public Choicen, in What Should Economist Do? Indianapolis, Liberty Press, 1979, p. 179. Souligné par I'auteur.

7 Barry, Norman, «The Liberal Constitution : Rational Design or Evolution?», in Critical Review, vol. 3, 1989, p. 268. 
politicien, le bureaucrate y apparaissent sous les traits de l'homo œconomicus : I'activité politique, c'est-à-dire l'activité d'individus agissant collectivement plutôt qu'individuellement, $y$ est perçue comme une forme d'échange, analogue à l'activité économique de ces individus, plutôt que comme la poursuite d'une vérité ou d'un "bien commun». Cette analyse économique de la politique présente la croissance du Léviathan moderne comme une conséquence non voulue des actions rationnelles des individus : pour Buchanan, Léviathan, c'est nous. La situation se complique toutefois lorsqu'on cherche à passer de l'analyse positive aux recommandations normatives, à faire de ce qui s'est avéré une représentation commode de la réalité un guide pour la conduite politique. Pour respecter son ambition d'un libéralisme qui ne nécessite pas la convergence sur des fins, Buchanan se doit de postuler que toutes les préférences ont une égale valeur et de professer à cet égard un agnosticisme radical. II se doit aussi de poser l'existence d'un consensus comme seul critère d'appréciation. Croit-on, par exemple, que des individus incertains de leur position future consentiraient à la création d'un important déficit budgétaire, sachant qu'ils ont, disons, une chance sur deux de se retrouver dans la génération qui aura à rembourser cette dette plutôt que dans celle qui profitera de l'emprunt $^{8}$ ? Mais autant le contractualisme de Buchanan peut nous amener à conclure que bien des règles actuellement en vigueur échoueraient au test de l'unanimité, autant ce même test d'unanimité interdit à Buchanan de proposer que d'autres arrangements, plus libres suivant les canons d'un libéralisme auquel Buchanan lui-même adhère subjectivement, puissent être implantés sans que les individus touchés manifestent leur accord avec ces changements. Singulier libéralisme que celui où la plupart des éléments du credo classique sont ravalés au modeste rang de préférences personnelles! En raison de cette exigence astreignante, Buchanan s'est d'ailleurs vu reprocher de faire l'apologie du statu quo. Reconnaissons toutefois que ce scepticisme, gênant à première vue, a l'avantage de rendre d'autant plus crédibles le diagnostic sévère que pose Buchanan sur les arrangements actuels de l'État-providence et la déontologie qu'il nous prescrit si nous

8 "The Constitution of Economic Policy", p. 313. II n'est pas inintéressant de savoir que Buchanan fut membre, aux côtés notamment de Milton Friedman, du Tax Reduction Task Force, mis sur pied par Ronald Reagan alors que ce dernier était gouverneur de Californie. Voir Adams, J.R. , Secrets of the Tax Revolt, New York, Harcourt Brace Jovanovich, 1984, p. 160. 
souhaitons «de libres relations entre des hommes libres». Alors que l'avènement de la Cité libérale favorisée par Hayek ou celui de l'utopie prêchée par les anarcho-capitalistes dépendent d'une conversion des esprits à leurs vertus supérieures, Buchanan propose de procéder par voie d'échanges, c'est-à-dire sans faire abstraction des individus tels qu'ils sont et sans faire violence à leur volonté. C'est justement parce qu'une société plus libre doit être librement choisie qu'on ne peut à l'avance en dessiner les formes exactes. Toute l'ambition de Buchanan est de formuler un libéralisme exclusivement fondé sur des critères méthodologiques et de se dispenser ainsi d'invoquer des valeurs dont il ne peut démontrer la supériorité de façon irréfutable. Mais peut-on, lorsqu'on fixe des normes, fussent-elles purement procédurales, se soustraire aussi aisément à ses propres préférences? Peut-on, en arguant de la possibilité d'un accord sur la méthode, échapper à la nécessité de choisir entre les fins?

L'objectif de cet article est de démontrer que Buchanan n'arrive pas à tenir son pari d'un libéralisme purement méthodologique. Pour ce faire, eu égard à la position "archimédéenne» qu'occupe l'individualisme méthodologique dans l'ensemble de sa construction, nous commencerons par examiner l'argumentaire invoqué par Buchanan pour établir la supériorité de cette approche. Dans un deuxième temps, nous analyserons les principaux concepts qui forment l'ossature de ce que Buchanan appelle tantôt son projet de "révolution constitutionnelle», tantôt, en termes moins militants, son approche contractualiste. Cet examen devrait nous permettre d'établir que sa défense du statu quo ne s'explique pas uniquement par le scepticisme moral, mais aussi par la crainte que la mise en œuvre de changements ne faisant pas consensus ne mine la stabilité du gouvernement et ne conduise à un état d'anarchie encore plus indésirable. II s'agira donc essentiellement $d^{\prime}$ un exercice relevant de la critique interne et qui portera non pas sur la substance des changements politiques proposés par Buchanan, par exemple en matière de politique fiscale, mais plutôt sur l'architecture intellectuelle de son argumentation.

\section{Vertus de l'individualisme méthodologique}

Tout ce qu'exige l'individualisme méthodologique, dit Buchanan, $c$ 'est que l'on reconnaisse que les individus poursuivent des fins et que ces fins sont diverses. Cette diversité des préférences est pour lui un donné : le fardeau de la preuve repose 
sur celui qui, à l'encontre de l'observation empirique, postule une identité des préférences. Considérer les individus comme les unités de prise de décision (decision-making units) tant dans leurs activités collectives que dans leurs activités privées, c'est donc faire appel à des postulats fort simples et difficilement contestables. En revanche, les modèles non individualistes se heurtent à un certain nombre d'objections. Celui auquel Buchanan compare le plus souvent l'approche individualiste, $c^{\prime}$ est le modèle organiciste où «l'État est considéré comme une unité de prise de décision unique agissant pour la société dans son ensemble ${ }^{9}$ ". Mais si les individus, comme l'affirme la conception individualiste, prennent des décisions en fonction de leur propre échelle de préférences, en fonction de quelles préférences un acteur collectif choisit-il? Pour résoudre ce problème, les tenants des modèles non individualistes sont tenus de faire appel à l'idée d'un «intérêt public» distinct des préférences privées ou, s'ils sont économistes, de construire une "fonction d'utilité sociale». Or, le célèbre théorème d'Arrow démontre qu'il est impossible de définir une telle fonction, en raison de l'intransitivité des préférences agrégées ${ }^{10}$. Quant à l'intérêt public, ce "Saint-Graal» de la politique, sa définition est hautement problématique. D'où tirerait-il sa source sinon des préférences et des valeurs individuelles? L'idée même d'un intérêt public, objet de l'activité politique, supposant celle d'un intérêt privé, objet de l'activité économique, comment expliquer que dans un cas l'on postule l'identité des préférences, tandis que dans l'autre on admet leur altérité? Telles sont quelques-unes des apories auxquelles conduit une approche non individualiste de la politique. Le point de vue individualiste est pour sa part plus économique et plus cohérent : il n'a pas besoin de recourir à des préférences ou à des valeurs autres que celles qui motivent les actions des individus; il pose que ceux-ci ne se comportent pas différemment, qu'ils agissent individuellement ou collectivement.

$2 \quad$ "The Pure Theory of Government Finance", in Fiscal Theory and Political Economy, Chapel Hill, University of North Carolina Press, 1960, p. 9.

10 Plus précisément, il est impossible de définir cette fonction sans violer au moins I'une des conditions posées par Arrow. Un dictateur peut bien sûr construire une fonction d'utilité sociale en imposant ses propres préférences. Sur ce point, voir : Arrow, Kenneth, Social Choice and Individual Values, New York, Wiley, 1963 [1951], ch. V. 
L'individualisme méthodologique permet par ailleurs de ramener les phénomènes politiques à leurs unités constitutives élémentaires, c'est-à-dire les individus. La perspective individualiste suppose que la politique est une activité par laquelle les individus tentent d'obtenir collectivement des biens qu'ils ne peuvent se procurer individuellement, par l'échange marchand. On pourrait parler de réalisme dans le sens où la représentation des processus politiques suggérée ici correspond mieux à la réalité empirique que ne le font les approches qui prennent pour unités constitutives des groupes, des organisations ou l'État lui-même. Toutes ces approches ont en effet pour point commun de voir en l'État un "despote bienfaisant". Muni de quelques hypothèses relatives au comportement prévisible du politicien, l'individualisme méthodologique est pour sa part en mesure de comprendre, par exemple, la faillite des politiques fiscales inspirées de la théorie keynésienne et adoptées par la plupart des pays occidentaux au lendemain de la guerre. La prescription suggérée voulait, on le sait, que dans les périodes de dépression, un accroissement des dépenses publiques restaure le plein emploi et la prospérité, tandis que devant la menace de l'inflation, on cherche plutôt à constituer des surplus budgétaires ${ }^{11}$. Pour les tenants de cette médecine, le gouvernement n'avait qu'à suivre ces conseils, et l'instabilité économique serait ainsi contenue. Mais raisonner ainsi, dit Buchanan, c'était adopter une image «irréaliste, naïve, a-historique et utopique" du gouvernement, c'était concevoir celui-ci comme un "despote bienfaisant ${ }^{12}$ ". En effet, seul un régime autoritaire pourrait mettre en œuvre les prescriptions susdites, parce que si la logique électorale des démocraties n'empêche pas - bien au contraire - la création de déficits budgétaires, on ne voit pas en revanche comment elle peut inciter politiciens et partis à constituer

1 Buchanan, James M., Burton, John et Richard E. Wagner, The Consequences of Mr Keynes, Londres, Institute of Economic Affairs, 1978, p. 15. II s'agit ici de Keynes tel que l'interprète Buchanan. Ce qui nous interesse, c'est le type d'explication plutôt que la justesse de cette explication.

12 "This 'benevolent despot' designation is convenient because economists have placed themselves in positions of proffering advice on policy reform, as if the political decision-makers, whoever these may be, will accept and act on such advice in total independence from any human motivations or impulses that political decision-makers themselves might possess". Brennan, Geoffrey et James M. Buchanan, Monopoly in Money and Inflation, Londres, Institute of Economic Affairs, 1981, p. 19. 
des surplus ${ }^{13}$. En négligeant de tenir compte du cadre dans lequel sont prises les décisions politiques, les avocats du "fine-tuning" ont proposé une politique qui, en dépit de leurs voux, conduisait à des déficits chroniques et croissants.

Au-delà de la simplicité et du réalisme, un troisième argument avancé par Buchanan en faveur de l'individualisme méthodologique tient à ce que celui-ci nous prédispose à la neutralité axiologique mieux que ne le font les autres approches. Alors que le tenant de l'existence d'un intérêt public consacre une bonne partie de ses efforts à définir cet intérêt et donc à persuader de ce qui devrait être, l'individualiste, ayant admis au départ la diversité des préférences et l'impossibilité d'en faire dériver un bien commun, peut consacrer ses énergies à la description de ce qui est. Certes, l'individualiste n'est pas hors de ce monde, il ne transcende pas les intérêts et les passions, mais, réalisant "qu'il n'est qu' un parmi plusieurs", il peut plus facilement faire preuve de "I'humilité que demande la science ${ }^{14}$ ". Dans The Calculus of Consent, Buchanan et son coauteur, G. Tullock, vont même jusqu'à écrire qu'on pourrait tirer des conclusions collectivistes d'une analyse individualiste tout aussi bien que des normes individualistes d'une analyse collectiviste ${ }^{15}$. Le moins qu'on puisse dire, c'est que cette césure est moins apparente dans les écrits postérieurs de Buchanan. Comment ne pas voir la parenté entre la profession de foi kantienne évoquée plus haut et l'idée suivant laquelle l'adhésion à l'individualisme méthodologique implique eque nous devrions traiter l'individu humain comme I'entité philosophique de base ${ }^{16} "$ ? Ainsi, la vision contractualiste, qui, du consentement individuel, fait dériver l'existence de l'État, pourra s'autoriser de l'individualisme méthodologique. De la même façon, le refus d'imposer des changements non désirés (ce que les critiques de Buchanan appellent son apologie du statu quol apparaîtra comme une conséquence de ce postulat démocratico-égalitaire : "chaque homme compte pour un et $c^{\prime}$ est

13 Ibid., p. 19-20.

14 The Limits of Liberty, p. 2.

15 Buchanan, James M. et Gordon Tullock, The Calculus of Consent, Ann Arbor, The University of Michigan Press, 1962, p. 315-317.

16 «An Individualistic Theory of Political Process», in Economics : Between Moral Philosophy and Predictive Science, p. 224. 
tout ${ }^{17}$ ". Et c'est enfin «simplement à titre de conséquence nécessaire d'une méthodologie individualiste-démocratique que la liberté individuelle devient l'objectif suprême de la politique sociale $^{18}$ ".

Ce passage de l'individualisme méthodologique à l'individualisme normatif appelle deux remarques.

Première remarque. La défense de propositions normatives au moyen d'un argument de type méthodologique présente des avantages certains sur le plan rhétorique. D'une part, le caractère normatif de la proposition avancée s'en trouve atténué, puisque celle-ci apparaît comme la conclusion obligée d'une position méthodologique. D'autre part, on immunise ainsi la proposition normative, sa réfutation passant par celle, plus difficile, de la position méthodologique dont on prétend la faire découler. On peut dès lors prétendre éviter, ou tout au moins repousser, la discussion sur les fins et les valeurs. Voilà un exemple clair d'effet idéologique généré par le recours à l'individualisme méthodologique : les vertus heuristiques qu'on associe à cette approche rejaillissent tout naturellement sur les conclusions normatives de ceux qui s'en font les champions et leur confèrent une apparence d'évidence qu'elles n'auraient pas autrement ${ }^{19}$.

Seconde remarque. Si cet effet peut s'exercer, en d'autres termes si le lien entre l'individualisme méthodologique de Buchanan et son contractualisme n'apparaît pas trop distendu, c'est en raison du caractère formel de celui-ci et de l'indétermination de son contenu. Pour bénéficier d'une telle caution méthodologique, le

17 The Limits of Liberty, p. 2.

18 Ibid.

18 Nous empruntons cette notion d'effet idéologique à Maurice Lagueux. Dans «Le néo-libéralisme et la gauche» lin Beaudry, Lucille et Lizette Jalbert, dir., Les métamorphoses de la pensée libérale. Sur le néo-liberalisme actuel, Sillery, Presses de l'Université du Québec, 1988), celui-ci attire l'attention sur * $\left(l^{\prime}\right)$ équivoque $(. .$.$) qui contribue au role idéologique de l'idée que véhicule la$ «main invisible» de Smith ou les unintended consequences de Hayek». II ajoute : * (...) cette idée me paraît associée à une contribution scientifique importante qui (fournit) la clé indispensable à toute explication de ces mécanismes que sont les divers marchés. Mais, il n'y a aucune raison pour que la qualité de l'explication rejaillisse sur la chose expliquée. L'analyse d'une concertation consciente des agents économiques n'offre pas autant d'intérêt théorique que celle de ce mécanisme inconscient, mais ceci $n$ 'implique naturellement pas qu'une telle concertation ne puisse produire des résultats économiques plus satisfaisants». 
libéralisme de Buchanan se doit d'être agnostique. Toutefois, on ne s'étonnera pas qu'à cet agnosticisme quant au contenu du contrat social, réponde une défense vigoureuse de la perspective méthodologique, laquelle permet d'établir l'idée d'un tel contrat. Certes, Buchanan se montre parfois conciliant et admet que le modèle individualiste n'est qu'un parmi plusieurs, mais sa défense de l'individualisme méthodologique est en général beaucoup plus vigoureuse. Pour lui, ceux qui refusent de voir dans les relations entre classes, États et organisations des phénomènes d'abord individuels renoncent par le fait même à une véritable science sociale ${ }^{20}$.

\section{Le contrat social}

Pour Hayek, c'est avant tout l'idéologie qui explique le développement de l'État-providence. Obnubilés par un idéal égalitaire, intellectuels et politiques ont, selon lui, cherché à rectifier ce qu'ils percevaient comme des injustices distributives résultant de l'ordre spontané du marché. Or, il n'existe pas de moment de la distribution distinct de celui de la production (c'est la grande erreur de Mill que d'avoir théorisé une telle dichotomie), et les interférences de l'autorité publique dans un ordre aussi complexe ne pouvaient dès lors que générer des conséquences indésirables (c'est le grand mérite d'un Bastiat d'avoir prévenu des

20 «This statement (i.e. that political process can be "factored down" to the level of individual choices) need not, of course, involve the claim of exclusive, or even predominant, relevance for the individualistic model in making predictions about political decision processes. In the scientific house there are many mansions, and, in analyzing politics, there is surely room for alternative models. For some purposes, an organic model may be helpful; for others, a ruling-class or force theory of the state». «An Individualistic Theory of Political Process", p. 224. Notons que ce texte est paru d'abord dans Easton, David, (éd.), Varieties of Political Theory, 1966, un ouvrage dont le titre indique la vocation œcuménique. La pensée de Buchanan sur les mérites particuliers de l'approche individualiste nous semble mieux traduite par cette déclaration prononcée lors du colloque sur les paradigmes en sciences sociales, tenu a l'université de Chicago en 1986 : *Those who prefer to conduct inquiry into the relationships among classes, states and other organizations as such, and without attempts to reduce analysis to the individuals who participate, do not, in my view, pass muster as social scientists in any useful sense of the term". Cité dans Hirsch, Paul, Michaels, Stuart et Ray Friedman, "'Dirty Hands' versus 'Clean Models': Is Sociology in Danger of Being Seduced by Economics?", Theory and Society, 1987, vol. 16, p. 317. Souligné par les auteurs. A cette époque, Buchanan était bien sâr au summum de sa carrière. 
dangers d'une politique menée à coups d'expédients plutôt qu'en conformité avec des règles). Une dérive idéologique appelant un remède du même type et le retour à une société authentiquement libre passent nécessairement par un renoncement au «mirage de la justice sociale" et un enthousiasme renouvelé pour les idées libérales classiques. On trouve une analyse du même type dans les écrits de Milton Friedman : c'est "le mythe de l'égalité des résultats" qui est à l'origine de nos déboires. Comme Hayek, il opte pour la stratégie du prosélytisme, confiant dans la supériorité intrinsèque de l'idéal qu'il propose.

La démarche de Buchanan est différente. Comme Hayek et Friedman, il regrette que nous ayons perdu cette sage crainte qu'éprouvaient les philosophes du XVIII' siècle à l'endroit du gouvernement. $\dot{A}$ leur instar, il lui arrive de décrire I'État-providence comme une accumulation d'erreurs ${ }^{21}$. Mais le retour à l'état de nature tel que le proposent les anarcho-capitalistes lui paraît une utopie idyllique ${ }^{22}$. Si des individus vivant dans l'inquiétude permanente ont intérêt à désarmer et pour cela à convenir des termes d'une entente, celle-ci ne pourra durer que si tous s'accordent pour désigner un arbitre chargé de la faire respecter et de punir les contrevenants. En l'absence d'un tel arbitre, chacun sera incité à la défection dès qu'il aura le sentiment que sa position s'est renforcée : ce qu'on croyait être la paix n'aura été qu'une trêve. Le gouvernement est donc pour Buchanan une nécessité, et l'on peut considérer que son existence dérive du consentement individue ${ }^{23}$. Mais une fois que le gouvernement existe, "le génie ne peut plus rentrer dans la

21 "The modern welfare state represents the mistakes of almost a century". "Dismantling the Welfare State", in Liberty, Market and State. Political Economy in the 80s, New York, New York University Press, 1985, p. 182.

22 «The libertarian anarchists who dream of markets without states are romantic fools, who have read neither Hobbes nor history". "Retrospect and Prospect", in What Should Economists Do?, p. 282.

23 \&Individuals will be led, by their own evaluation of alternative prospects, to establish by unanimous agreement a collectivity, or polity, charged with the performance of specific functions, including, first, the provision of the services of the protective or minimal state and, second, the possible provision of genuinely collective consumption services». Brennan, Geoffrey et James M. Buchanan, The Reason of Rules, p. 22. 
bouteille", la croissance du Léviathan moderne est désormais possible. En toute rationalité, les individus adopteront des règles qui les conduiront à effectuer des choix incohérents; des décisions qui, prises séparément, répondent aux désirs des individus déboucheront, une fois agrégées, sur des résultats que personne ne souhaitait; des mécanismes de représentation constituant une réponse tout à fait justifiée à des coûts de décision trop élevés introduiront une diffraction dans la traduction des volontés individuelles. Buchanan en conclut : "When we speak of controlling Leviathan we should be referring to controlling self-government, not some instrument manipulated by the decision of others than ourselves ${ }^{24}$ ". C'est donc une version du contrat social, version consciente des dilemmes qui toujours guettent l'action collective, que nous propose Buchanan.

Ce contrat social constitue d'abord un outil diagnostique : une société donnée et les divers arrangements qui la caractérisent peuvent-ils être interprétés comme les résultats d'une délibération collective? Mais l'approche contractualiste prescrit également une méthode : toute réforme suggérée en vue de corriger les imperfections décelées devra elle aussi subir le même test. Si l'on estime que la valeur d'un arrangement tient non pas à un critère apodictique mais à ce qu'il recueille l'assentiment général, le meilleur arrangement sera celui dont les contractants conviendront. Cela implique que, pour Buchanan, la limite entre le secteur public et le secteur privé ne peut être tracée a priori ou transcrite d'un manuel d'économie libérale : elle passera là où les contractants en conviendront ${ }^{25}$. Le contrat social est "l'analogue politique de l'échange marchand" : tout comme la conclusion de celui-ci témoigne de la satisfaction des partenaires, un arrangement qui suscite l'assentiment unanime sera par définition supérieur à tout autre qui ne témoignerait pas d'un tel degré d'adhésion ${ }^{26}$. Au contraire de Hayek et de Friedman qui font de la conversion des esprits la voie vers une société plus libre, Buchanan mise sur l'échange générateur de gains mutuels : si une situation donnée est jugée insatisfaisante, la tâche de l'économie politique normative sera d'imaginer des arrangements nouveaux dont tous tireront bénéfice.

24 The Limits of Liberty, p. 149.

Ibid., p. 72.

26 *The Constitution of Economic Policy», p. 309. 
Pour Buchanan, en effet, la grande découverte de l'économie politique classique, son principe central, c'est l'idée suivant laquelle "les actions séparées des individus peuvent être coordonnées à travers une structure de marchés reliés entre eux et contenus dans un système légal-gouvernemental chargé de faire respecter les droits de propriété et les contrats ${ }^{27}$ ". L'analyse économique n'a donc pas pour objet les résultats de l'échange, mais bien le processus d'échange lui-même ${ }^{28}$. En adoptant la définition robbinsienne, aujourd'hui dominante, qui voit dans la science économique une discipline centrée sur l'allocation des ressources rares entre des fins concurrentes ${ }^{29}$, les économistes ont cependant été amenés à détourner leur attention des processus d'échange et à la porter sur les résultats. En devenant "spécialistes du choix", ils se faisaient du même coup ingénieurs sociaux et se vouaient à la recherche d'une fonction d'utilité sociale ou au calcul utilitariste de la solution optimale ${ }^{30}$. La distinction entre processus et résultats prend une signification encore plus nette lorsqu'on se situe sur le plan strictement normatif. Pour le libéral classique, la justice d'une répartition donnée ne peut être établie par le seul examen de son contenu; elle dépend plutôt de la façon dont cette répartition est advenue. Pour le partisan de la «justice sociale», le contenu ou le résultat sont au contraire déterminants. C'est pourquoi il s'autorise, par exemple, à décréter que des profits sont uexcessifs", sans égard à la manière, frauduleuse ou légale, dont ils ont été obtenus.

Si l'on applique cette conception échangiste au domaine de la politique, c'est la théorie du contrat social qui offre le cadre le plus approprié. Certes, l'échange politique est conceptuellement et pratiquement plus complexe que l'échange économique. D'une part, le nombre de participants y est plus élevé : à la différence de l'échange économique qui, dans sa version idéaltypique, n'implique

27 "Political Economy and Social Philosophy", in Liberty, Market and State, p. 267.

28 "What Should Economists Do?", in What Should Economists Do?, p. $30-31$.

29 «Economics is the science which studies human behaviour as a relationship between ends and scarce means which have alternative uses". Voir Robbins, Lionel, An Essay on the Nature and Significance of Economic Science, New York, New York University Press, 1984 [1932], p. 16.

30 «Political Economy and Social Philosophyn, p. 262-267. 
que deux personnes, il réunit tous les membres de la collectivité. D'autre part, l'échange politique doit avoir pour conclusion une décision qui s'appliquera à tous. L'échange économique est beaucoup plus simple, puisque la transaction y porte sur des biens différents et sur des quantités différentes de ces biens. Exiger que les membres de la collectivité se prononcent à l'unanimité sur chacune des décisions les concernant reviendrait à faire porter la discussion sur les résultats. Or, comment des individus dont les préférences diffèrent parviendront-ils à s'entendre? Si l'on recherche l'équivalent politique de la distinction tracée plus haut, on devra renoncer à ce que le contrat ait pour objet un état final jugé supérieur et devant se perpétuer. II faudra donc distinguer entre le choix que l'on peut faire parmi diverses règles et celui que I'on peut faire entre diverses options au moyen de ces règles. C'est le premier terme de cette alternative, et non le second, qui devra être porté devant les contractants. II serait en effet utopique d'exiger que chaque décision recueille l'adhésion de tous : les résultats de cette décision étant largement prévisibles, les intérêts divergents des individus et des groupes poseraient un obstacle insurmontable à la recherche d'un consensus ${ }^{31}$. En revanche, un accord sur le type de décisions que l'on peut prendre collectivement ou sur la majorité requise pour que tel ou tel type de décision soit pris paraît possible. Les règles d'un jeu sont en effet indépendantes du déroulement d'une partie donnée et, même si cette indépendance est moins nette dans le jeu de la politique, on peut affirmer que "le champ pour une entente possible sur des règles est nécessairement plus large que celui pour un accord sur des résultats découlant de l'application de règles précises ${ }^{32}$ ». Ce qui permet justement un accord sur des règles, c'est qu'au moment d'en convenir, les contractants ne peuvent prédire si les décisions qui se prendront au moyen de ces règles les avantageront personnellement ou non. Les individus ayant à s'entendre sur des règles communes se trouvent donc devant un voile d'incertitude. La possibilité de dégager un consensus est nettement liée à l'opacité de ce voile, et cette opacité est à son tour dépendante de la généralité et du caractère durable des règles qui seront discutées $^{33}$. Des individus ayant à choisir entre des règles plutôt

31

32

Brennan, Geoffrey et James M. Buchanan, The Reason of Rules, p. 29.

Ibid.

Ibid., p. 29-30. 
qu'entre des options précises, à partir desquelles il est aisé de prédire des résultats, auront donc tendance à accorder une place plus grande dans leurs délibérations à des critères d'équité susceptibles de généralisation ${ }^{34}$. Cette attitude apparemment altruiste ne tient cependant pas à l'angélisme des contractants. Elle procède au contraire de leur intérêt bien compris ${ }^{35}$. II est, par exemple, peu probable que des personnes qui, «au moment du contrat, ne sont pas en mesure de prédire leur propre position future $(. .$.$) conviennent de confier une autorité politique illimitée à$ quelque groupe que ce soit, qu'il s'agisse d'une majorité parlementaire dûment élue, $d$ 'une élite judiciaire ou d'une junte militaire ${ }^{36}$ ". À ce point, la distinction entre processus et résultats ainsi que son importance quant au contrat social devraient être claires.

II convient maintenant de quitter ce niveau abstrait et général pour examiner d'abord sur quoi doit porter concrètement le contrat, puis comment l'exigence d'unanimité peut trouver une application pratique dans un monde où elle n'est pas la règle.

\section{Contrat constitutionnel et contrat postconstitutionnel}

On peut distinguer conceptuellement le contrat constitutionnel du contrat postconstitutionnel. Le premier correspond au passage de l'état de nature à l'état de société : il porte sur les limites que s'imposent les individus dans leur comportement vis-à-vis des autres, sur les droits de propriété qu'ils se reconnaissent mutuellement ainsi que sur la façon dont le respect de ces limites et de ces droits sera assuré. La conclusion de ce contrat constitutionnel est marquée par l'émergence d'un État protecteur. Le contrat postconstitutionnel traite pour sa part des activités que les individus choisiront d'organiser collectivement. II devrait tracer une démarcation générale entre ce qui relève de l'initiative individuelle lou de la coopération

\footnotetext{
34 "The Constitution of Economic Policy", p. 310-311.

36 $\quad(\ldots)$ faced with genuine uncertainty about how his position will be affected by the operation of a particular rule, the individual is led by his self-interest calculus to concentrate on choice options that eliminate or minimize prospects for potentially disastrous results». Brennan, Geoffrey et James M. Buchanan, The Reason of Rules, p. 16.
}

36 «A Contractarian Perspective on Anarchy", in Freedom in Constitutional Contract, p. 16. 
volontaire) et ce qui nécessite la contribution de tous. II devrait également fixer les règles suivant lesquelles seront prises les décisions relatives à l'organisation des activités collectives et celles déterminant le mode de financement de ces activités. La forme institutionnelle de ce contrat est l'État producteur. Ce modèle contractualiste, rappelons-le, ne se veut en aucune façon la description de l'origine historique des sociétés réelles. Sa fonction est essentiellement d'évaluer dans quelle mesure les institutions existantes peuvent être légitimées et de fournir des critères aptes à suggérer des réformes là où l'on observe des situations qui ne sauraient résulter d'une entente entre individus rationnels ${ }^{37}$.

L'état de nature hobbesien constitue le point de départ de l'analyse : les individus y sont inégaux, tant dans leurs capacités que dans la quantité de biens qu'ils contrôlent. L'agression et l'insécurité y sont la règle. Il peut arriver toutefois que les personnes vivant dans cet état de nature atteignent ce que Buchanan appelle l'équilibre naturel, c'est-à-dire un point où tous perçoivent que la perpétuation de la guerre généralisée leur impose des coûts trop élevés et ont le sentiment qu'il existe peut-être un arrangement différent qui permettrait à tous d'améliorer leur condition. Ils choisiront dans ce cas de modifier leur comportement et instaureront une trêve (Buchanan parle d'un "accord de désarmement" $)^{38}$. Les conditions sont alors réunies pour qu'ait lieu une discussion sur la définition des droits de propriété de chacun ${ }^{39}$. Rien n'assure que la répartition sur laquelle les contractants s'entendront sera «juste", si cette justice est fonction d'un étalon extérieur ou transcendant. Au contraire, il est probable que l'entente reflétera en bonne partie le rapport de forces qui prévalait antérieurement. Mais que les parties concluent un tel accord témoigne de l'amélioration qu'il représente pour elles par rapport à la situation initiale. Si tel n'était pas le cas, il n'y aurait pas d'entente, car la partie qui s'estime lésée ne se sentirait nullement liée et romprait la trêve. Une fois que les contractants

37 Voir The Limits of Liberty, p. 50 et «Before Public Choice», p. 82.

38 The Limits of Liberty, p. 59.

39 Buchanan définit ainsi les droits de propriéte : "The positive rights of possession or domain over stocks of goods, or more generally, over resource endowments capable of producing final goods. These endowments include human capacities (the right to one's own person...), as well as nonhuman factors, including domain over territory». Ibid., p. 72. 
ont agréé la nouvelle répartition des droits de propriété, il est impérieux pour tous que chacun en respecte les termes et ne soit pas tenté de retourner à l'état de nature. Or, le respect de l'accord se heurte à un obstacle de taille : chacun, en effet, ne sera incité à respecter l'entente conclue que s'il a l'assurance que tous en feront autant ${ }^{40}$. II est alors parfaitement rationnel que les parties s'accordent sur la désignation d'un arbitre chargé de faire observer les règles du contrat et d'infliger des châtiments aux contrevenants ${ }^{41}$. L'émergence d'un État protecteur peut donc être interprétée comme le résultat du calcul rationnel $d$ 'individus inégaux vivant dans l'état de nature. Il est cependant important de noter que ce "saut hors de la jungle anarchique" dépend de l'atteinte d'un point d'équilibre préexistant au contrat et qui, lui, ne peut être justifié dans la perspective contractualiste ${ }^{42}$. C'est ici que l'agnosticisme moral de Buchanan est le plus évident : celui-ci refuse catégoriquement d'invoquer l'existence de droits naturels antérieurs au contrat pour porter jugement sur la répartition qui caractérise ce point d'équilibre et déterminera en bonne partie le contenu du contrat ${ }^{43}$. Il ne peut exister de droits antérieurs à ce contrat, puisque de tels droits ne pourraient alors se fonder que sur des justifications extérieures et supérieures aux individus en cause. Or, pour Buchanan, celui qui affirme l'existence de valeurs absolues ne fait ainsi qu'énoncer ses propres préférences et se

$40 \quad$ Ibid., p. 65.

41 Ibid., p. 67.

42 Ibid., p. 58.

43 Les transitions qui ont affecté les pays ex-socialistes peuvent être décrites dans les termes de ce modèle. A l'origine, on se trouve dans un état de nature, puisqu'un régime totalitaire ne saurait émerger d'un accord entre individus rationnels. Les parties (nous les réduirons, pour les besoins de la démonstration, à deux) ont cependant atteint un point d'équilibre naturel : le pouvoir est réticent à l'idée de mater ses adversaires par les armes, l'opposition ne veut pas recourir a la violence contre le pouvoir affaibli. Volontairement désarmées, les parties peuvent dès lors discuter de la répartition des droits de propriété. L'arrangement suivant est possible : les entreprises seront privatisées et les bureaucrates communistes, puisque ce sont eux qui disposent de la majeure partie de l'épargne, en deviendront plus souvent qu'autrement les acquerreurs. Cette nouvelle situation reflète certes l'ancien rapport de forces, mais elle pourrait être jugée préférable par les deux parties. Un accord en consacrerait la légitimité. 
soustrait par là au discours rationnel ${ }^{44}$. La situation initiale ne peut donc être dite juste ou injuste : dans l'état de nature, l'existence est malheureusement "solitary, poor, nasty, brutish and short", et le contrat permet justement d'échapper à cet environnement redoutable. II serait en revanche injuste qu'un des contractants se dérobe à la règle à laquelle il a acquiescé: toute modification du contrat suppose qu'à nouveau il y ait unanimité. Le critère qui permet de décréter la justice ou l'injustice d'une situation ne réside donc pas dans le contenu de celle-ci, mais plutôt dans la manière dont elle a vu le jour. En somme, le contrat social de Buchanan ne tire pas sa valeur des termes qu'il contient; c'est au contraire leur inscription dans un contrat qui donne valeur à ces termes.

La nécessité d'un État producteur et d'un contrat qui en délimite les fonctions procèdent du problème de l'action collective. La production de certains biens dont tous tireront avantage (l'assèchement d'un marais abritant des insectes nuisibles, pour reprendre un exemple cher à Buchanan et à Tullock) exige souvent, eu égard aux coûts qu'elle implique, un accord préalable de tous les membres de la collectivité. Or, dans une telle situation, il est tentant de ne pas révéler ses préférences et de vouloir profiter du bien collectif sans contribuer à sa production. Cette possibilité de resquillage met bien sûr en péril la production de tous les biens collectifs. Chacun n'acceptera de payer sa part que s'il a la garantie que tous paieront la leur. Jusqu'ici, la règle d'unanimité prévaut toujours : c'est en se réservant ainsi un droit de veto que chacun se protège contre une éventuelle obligation de contribuer au financement de biens dont il estime qu'ils ne lui procureraient pas des gains supérieurs aux coûts qu'il devra engager. Mais cette unanimité qui constitue, pour les biens collectifs, l'équivalent de l'échange entre deux personnes, pour les biens privés, impose des coûts énormes. Chacun se trouve dans une position stratégique lui permettant de marchander son consentement et risque de paralyser toute entente. Les contractants seront alors amenés à s'entendre sur une norme déterminant le nombre minimal d'adhésions nécessaire pour qu'une décision puisse s'appliquer à tous. En d'autres termes, ils se soumettront volontairement à la coercition. Toutefois, des individus rationnels n'accepteront pas de renoncer à leur droit de veto sans que soient adoptées en retour des

44 «Before Public Choicew in Freedom in Constitutional Contract, College Station, Texas A\&M University Press, 1977, p. 82-83. 
contraintes qui imposeront des limites à l'action collective et les protégeront d'une tyrannie de la majorité. Ce qu'on observe pourtant, c'est une érosion constante de ces contraintes constitutionnelles et une croissance concomitante des activités de l'État producteur. Cela tient au fait qu'étant nécessaire à la production de certains biens que le marché ne peut assurer de façon optimale (au premier chef, la sécurité), l'État apparaît comme la solution désignée à toutes les difficultés que pose la production volontaire d'autres biens et à ce que le financement des biens collectifs par l'impôt ou par l'emprunt amène les individus à tenir leur coût marginal pour nul et à ne pas compenser une demande accrue pour un type de bien collectif par une réduction de la demande pour un autre ${ }^{45}$.

Ce dénouement indésirable démontre, selon Buchanan, la nécessité d'une révolution constitutionnelle dont les éléments pourraient être l'adoption d'un seuil de décision plus élevé que la majorité simple et d'une constitution fiscale qui agirait comme un corset sur les dépenses de l'État ${ }^{46}$. Là encore, le comportement individuel peut être interprété au moyen de la théorie du choix rationnel : par un retour du balancier, l'adoption de réformes radicales apparaîtra préférable au maintien d'un "équilibre inefficace et surcollectivise $e^{47}$ ".

\section{Unanimité et statu quo}

On a reproché au principe de l'unanimité contractuelle d'être "essentiellement conservateur», de favoriser "la tyrannie du statu

$45 \quad \&(\ldots)$ the fact that publicly provided goods appear to be costless at the margin to the individual voter, makes it easier (more probable) to reach collective decisions that provide than ones that deny". Anthony de Jasay, "Is Limited Government Possible?», in Critical Review, 1989, vol. 3, p. 303-304. Souligné par I'auteur.

48 Parmi les mesures fiscales que prone Buchanan, mentionnons: la règle de l'équilibre budgétaire annuel, l'impôt proportionnel plutôt que progressif, des plafonds absolus aux taxes et aux dépenses. Voir Brennan, Geoffrey et James M. Buchanan, The Power to Tax: Analytical Foundations of a Fiscal Constitution, Cambridge, Cambridge University Press, 1980.

47 «Pragmatic Reform and Constitutional Revolution», in Freedom in Constitutional Contract, p. 275. 
quo ${ }^{48}$ ", donc de maintenir des imperfections que l'approche contractualiste avait justement pour fonction de déceler. Exiger le consentement unanime comme préalable au changement revient en effet à dire qu'il n'existe pas de droits absolus antérieurs au contrat et que, en l'absence d'un accord général, le statu quo est préférable au changement. Certes, le statu quo n'a pas pour Buchanan de valeur en soi : reconnaître sa primauté n'est en aucune manière acquiescer au bien-fondé de son contenu. Dans son argumentation relative au statu quo, Buchanan n'adopte pas l'attitude du conservateur (à la Michael Oakeshott) pour qui le connu imparfait et familier est d'emblée préférable à l'inconnu plus prometteur mais gros de dangers. Il fait plutôt appel à trois attitudes : le réalisme, l'agnosticisme et le respect de l'autodétermination individuelle. II commence par nous enjoindre de reconnaître que le statu quo existe et que lui seul existe ${ }^{49}$. Puis, il pose que les jugements sur tel ou tel arrangement social sont portés par des individus et que les normes au moyen desquelles ceux-ci évaluent les institutions ne sont pas a priori susceptibles d'universalisation. Je peux, par exemple, m'opposer à l'État-providence par intérêt personnel (j'estime qu'il me coûte trop cher pour ce que j'en retire) ou par préférence idéologique (je n'aime pas le collectivisme), mais il n'y a là rien qui me permette d'affirmer que ma vision est supérieure à celle de ses défenseurs ${ }^{50}$. En fait, seul un diagnostic unanime, parce qu'il témoignerait d'un accord intersubjectif, satisferait au critère d'universalité. Faire abstraction du statu quo, c'est glisser soit vers l'utopisme soit vers l'autoritarisme: puisque la supériorité d'une proposition tient à l'adhésion qu'elle rencontre et non à un critère indépendant, on a le choix entre partir du statu quo et imposer ses préférences. Cette primauté du statu quo et cette exigence d'unanimité tiennent même dans le cas d'arrangements qui ne bénéficient pas de la légitimité contractuelle. On peut fort bien juger que le «privilège" dont bénéficie un groupe quelconque a été acquis «indûment", mais cela n'autorise nullement à le déclarer soudainement caduc si ceux qui en tirent avantage y résistent. En effet, un changement imposé n'aurait aucune valeur contractuelle

48 Lemieux, Pierre, La souveraineté de I'individu, Paris, Presses universitaires de France, 1987, p. 99.

The Limits of Liberty, p. 78.

«Dismantling the Welfare State», p. 179. 
et ne serait donc pas plus justifiable que la situation qu'il est censé corriger. Présentée de cette manière, la position de Buchanan semble effectivement une consécration du statu quo: pourquoi ceux qui profitent d'une situation y renonceraient-ils? Ils le feront, explique Buchanan, si on leur propose un échange qui comporte des gains mutuels : "si une structure institutionnelle existante est authentiquement inefficace, il doit exister un moyen de changer ou de réformer des éléments de cette structure de façon à ce que toutes les personnes ou tous les groupes dans la société en bénéficient ${ }^{51}$ ". II donne l'exemple d'un tarif douanier établi depuis longtemps. On peut avoir plusieurs raisons d'abolir ou de réduire ce tarif (faire baisser les prix à la consommation, stimuler les exportations, etc.), mais on ne devrait y procéder qu'avec le consentement des industries qui en bénéficient au moment présent. Or, il est prévisible que celles-ci ne donneront leur accord qu'en échange d'une compensation pleine et entière : celle-ci devra alors être financée à même une taxe prélevée auprès de ceux qui profiteront de la réduction ou de l'abolition du tarif ${ }^{52}$. II s'agit là sans doute d'un prix élevé, dit Buchanan, mais le sentiment d'illégitimité que susciteraient des changements unilatéraux décidés par une majorité, sûre de son droit, imposerait à l'ordre social des coûts encore plus élevés ${ }^{53}$. L'individualisme n'est pas ici que méthodologique, il prend aussi un caractère nettement éthique, en ce qu'il interdit que des règles (mais pas nécessairement des décisions particulières) puissent $s$ 'imposer aux individus sans leur consentement.

On a vu par ailleurs que Buchanan distinguait l'unanimité conceptuelle de la forme pratique qu'elle doit adopter lorsqu'une collectivité ou ses représentants ont à prendre une décision. L'unanimité conceptuelle est une norme dont doit tenir compte l'économiste politique. Son hypothèse de départ est que tout échange, qu'il soit politique ou économique, comporte des gains mutuels. Cela implique que si une proposition favorable à la majorité risque de désavantager une minorité en regard de sa situation présente, cette minorité doit être compensée pour la perte encourue. Seule une telle compensation pourrait l'inciter à donner

61 Ibid. Souligné par l'auteur.

62 «Positive Economics, Welfare Economics and Political Economy", in Fiscal Theory and Political Economy, p. 123-124.

63 «Dismantling the Welfare Statew, p. 184. 
son accord à la réforme proposée. Mais comment passer d'une unanimité conceptuelle, où l'on postule que tous les participants sont raisonnables, au monde réel, où ils ne le sont pas toujours? Comme nous l'avons mentionné, si l'unanimité est requise pour procéder à tout changement, chaque individu sera tenté de vendre son consentement au prix fort. L'économiste suédois Knut Wicksell, à qui Buchanan a emprunté le critère d'unanimité, voyait en celui-ci un garde-fou pouvant contenir les dépenses inappropriées de l'État et protéger les individus contre une contribution forcée à des activités contraires à leurs intérêts. II reconnaissait toutefois le risque de paralysie qu'il comportait ${ }^{54}$. On peut penser que des contractants rationnels se rendraient compte des coûts élevés de l'unanimité et l'on observe effectivement que les seuils de consentement requis pour qu'une décision politique soit prise lui sont inférieurs. Mais, dit Buchanan, il existe un fétichisme de la règle de majorité (plusieurs confondent majority rule et démocratie) dont les effets sont souvent indésirables : "le passage, dans la législature, de la majorité simple à une forme de majorité renforcée», apparaît donc comme une réforme éminemment désirable ${ }^{55}$. Ce faisant, l'on accorde certes une prime au statu quo, mais on protège en même temps les minorités contre des changements qu'elles ne souhaiteraient pas.

\section{Un libéralisme purement méthodologique?}

Ce que l'on peut se demander, par contre, c'est si Buchanan s'en tient effectivement à l'agnosticisme qu'il professe d'adopter, en d'autres termes s'il ne fonde pas ultimement sa déontologie sur un critère axiologique. La force de sa position, on l'a vu, tient à ce qu'il présente les règles de son contractualisme comme étant neutres sur le plan axiologique et directement déduites de ses prémisses méthodologiques. S'il peut procéder ainsi avec assez d'aisance, c'est qu'il insiste constamment sur la forme plutôt que

54 "Justification of the Compound Republic, The Calculus in Retrospect", in Gwartney, James D. et Richard E. Wagner, éd., Public Choice and Constitutional Economics, Greenwich, Conn, JAl Press, 1988, p. 134. Buchanan ajoute : «By reducing the requirement to, say, five-sixths of the voting members of the assembly, the incentives for strategic behavior are dramatically reduced and there is insurance against most, if not all, inefficient outlay". Ibid.

56 Tullock, Gordon, "The Calculus : Postcript After 25 Years», in Gwartney, James D. et Richard E. Wagner, éd., Public Choice and Constitutional Economics, p. 141. 
sur le fond, sur la méthode suivant laquelle se prennent les décisions plutôt que sur leur contenu, sur l'idée du contrat plutôt que sur la teneur de ses clauses. Le principal reproche qu'il adresse à John Rawls n'est-il pas d'être allé au-delà de ce qu'autorise strictement l'approche contractualiste en précisant le contenu des principes de justice auxquels devraient acquiescer des individus rationnels ${ }^{56}$ ? Buchanan semble effectivement se situer aux antipodes de ceux qui se proposent de définir ce qu'est le bien commun. II refuse obstinément de reconnaître l'existence de droits naturels qui seraient préalables au contrat et pourraient servir à l'évaluer. Il ne voit dans toute discussion sur les fins qu'une retraite «vers des débats stériles qui ont trait aux valeurs personnelles et où la discussion rationnelle ne joue plus aucun rôle" ${ }^{57}$. Pour lui, «il n'y a pas de normes transcendantes, suprapersonnelles ${ }^{58}$ ", l'autonomie axiologique des individus ne saurait être transgressée. Plusieurs sont perplexes devant le refus qu'il oppose à des réformes qui ne feraient pas le consensus et devant son insistance à tenir pour «illibéraux" des changements imposés, aussi conformes soient-ils aux canons de l'orthodoxie libérale. Ce "scepticisme moral hobbesien" a d'ailleurs valu à Buchanan bien des reproches. Ainsi, on a dit de sa démarche qu'elle revenait à "consacrer l'adage nihiliste might makes right ${ }^{59}$. L'exigence d'unanimité accordant un poids égal à toutes les préférences individuelles, on s'est interrogé sur le bien-fondé de traiter sur le même pied préférences sociales et antisociales ${ }^{60}$. On s'est également demandé pourquoi un libéral convaincu de la supériorité intrinsèque de certaines valeurs accepterait de voir le

56 The Limits of Liberty, p. 175.

67 «Before Public Choice», p. 82-83.

68 «Democratic Values in Taxation», p. 244.

59 Krauss, Michael, «Réflexions sur le contractualisme et les droits fondamentaux", communication présentée au colloque du Liberty Fund tenu à Montréal les 30 juin, 1er, 2 et 3 juillet 1988, p. 52 .

60 «Should we not discount some immoral and anti-social preferences (for example, those of the rapist, arsonist and sadist, and, less dramatically, of the envious)? Buchanan apparently does not want to do so, but this seems implausible». Hannes H. Gissurarson, «lndividual Rights and Spontaneous Order", communication présentée au colloque du Liberty Fund, p. 12. 
statut de celles-ci dépendre de l'accord de tous ${ }^{61}$. Mais ces critiques ont en commun de juger le point de vue de Buchanan à partir d'une position postulant l'existence de droits naturels ou la supériorité du credo libéral classique. Or, c'est là une position que Buchanan exclut au départ. Pour nous, il ne s'agit pas de trancher entre le scepticisme moral de Buchanan et la certitude de ses critiques. L'existence d'une éthique objective ne nous apparaît pas d'une évidence aveuglante et le subjectivisme extrême de Buchanan, certes contestable, ne nous semble ni plus inquiétant ni plus condamnable que l'aisance avec laquelle certains tenants des droits naturels résolvent les dilemmes les plus déchirants et les plus complexes. Si sa profession d'agnosticisme équivalait à la reconnaissance de la dichotomie classique entre faits et valeurs et si, en affirmant l'impossibilité de s'entendre sur une définition positive et indiscutable du bien commun, Buchanan se contentait de dire que la science ne nous délivre pas de la nécessité de choisir, on ne pourrait pas lui reprocher grand-chose. La thèse de la neutralité axiologique est certes controversée, mais, du moins dans son acception weberienne, elle a le mérite d'interdire le travestissement d'idéaux en conclusions scientifiques. Ce n'est toutefois pas à cette thèse que s'apparente la position de Buchanan. Pour lui, l'accord sur les fins est certes exclu, mais pour échapper au conflit des valeurs ultimes, il propose de faire porter la discussion sur des règles générales plutôt que sur des résultats. Or, l'impossibilité de prédire la position finale d'un individu au moment du choix des règles érige un écran entre celles-ci et l'état final correspondant aux préférences des individus. Par conséquent, la détermination de ces règles devient affaire de méthode. Buchanan écrit par exemple :

We can talk meaningfully about the "best" rules, or the " $n^{\text {th }}$ best" arrangements, often quite independently of the ultimate policy targets. In other words, we can talk normatively about "process" or "procedure", while staying clear of normative discussion of "end-states" ${ }^{62}$.

B1 Barry, Norman, «Unanimity, Agreement and Liberalism : A Critique of James Buchanan's Social Philosophy», Political Theory, 1984, vol. 12, p. 581. 
II deviendrait donc possible de faire dériver un "devoir-être» (ought), ou du moins un devoir-être d'un type particulièrement formel, d'un "être" (is), parce que ce devoir-être demeurerait fondamentalement indépendant ou, à tout le moins, passablement distinct des fins ultimes que poursuivent les individus ${ }^{63}$. En partant de la position méthodologique de l'individualisme, on pourrait formuler les meilleures règles, sans pour autant violer le principe de neutralité axiologique. Peut-on ainsi fonder des normes sociales sur une méthode ? Buchanan n'exige-t-il pas de l'individualisme méthodologique plus que celui-ci ne saurait offrir ?

Considérons des cas où une collectivité renégocierait le contrat social ou certaines de ses clauses. Imaginons qu'une majorité souhaite abolir les tarifs protectionnistes dont bénéficient certaines industries ou qu'elle veuille mettre fin à ce qu'elle estime être un privilège dont jouissent certaines catégories d'individus (par exemple, la sécurité d'emploi des professeurs d'université). On peut aussi penser, cas plus dramatique, à une société où l'émancipation d'une minorité en esclavage est souhaitée par une faible majorité des hommes libres. Dans chacun de ces cas, Buchanan nous interdit de procéder au changement désiré par le recours à la majorité s'il n'y a pas consensus sur la désirabilité du changement et sur les compensations qui devront être versées à ceux qui se sentiront lésés par ce changement. La position des industries favorisées ou des professeurs d'université doit être envisagée comme si elle résultait d'un contrat implicite entre ces groupes et le reste de la collectivité, contrat que l'on ne pourrait rompre unilatéralement. Même dans le cas des maîtres et des esclaves, dont on pourrait pourtant dire qu'ils vivent dans l'état de nature, il faut procéder comme s'il y avait eu entre eux entente préalable, puisque le contrat étant l'unique source du droit, la seule façon de fonder la légitimité des changements est de prendre le statu quo pour point de départ ${ }^{84}$. L'approche contractualiste de Buchanan oblige à obtenir l'adhésion des industries, des professeurs ou des propriétaires d'esclaves en leur offrant une compensation. Ce recours à l'exigence d'unanimité procède, selon Buchanan, non d'un choix axiologique, mais d'une de ses prémisses méthodologiques, c'est-à-dire la reconnaissance de la

63 Dériver un ought d'un is est le principal reproche que Scott Gordon adresse a Buchanan. Voir *The New Contractarians», in Journal of Political Economy, 1976, vol. 84, p. 573-590.

The Limits of Liberty, p. 75. 
diversité des préférences individuelles. Comme il n'existe pas de normes transcendantes permettant de déterminer d'une manière indubitable la justice ou l'injustice d'un état donné, c'est le désaccord entre les individus qui indique la nécessité de renégocier le contrat, et c'est leur unanimité seule qui peut révéler la possibilité d'un état supérieur. En d'autres termes, le consensus supplée à l'absence d'étalon suprapersonnel à partir duquel on pourrait évaluer le changement proposé.

II est clair cependant que Buchanan ne s'en tient pas à cet usage "épistémique»" ${ }^{65}$ du critère d'unanimité. Les situations évoquées ne sont pas sans contenu axiologique : les règles que certains sociétaires veulent changer traduisent le caractère dominant de certaines préférences sur d'autres. Prenons l'exemple d'une société dont une partie est tenue dans l'esclavage (situation A). On peut imaginer deux autres états possibles : celui où les esclaves sont libérés sans compensation pour les maîtres (situation B) et celui où l'émancipation résulte d'un accord s'accompagnant $d^{\prime}$ une pleine compensation (situation $\mathrm{C}$ ). Certes, dans un monde sans valeurs transcendantes, la simple substitution de $B$ à $A$ ne peut être tenue pour moralement supérieure au maintien de $A$, puisqu'elle ne résulte pas d'un accord unanime. En effet, si les preférences individuelles sont inviolables, seule la situation d'unanimité $(C)$ sera supérieure à toute situation de non-unanimité. Mais si ce point doit être concédé à Buchanan, on ne voit pas, en revanche, comment celui-ci peut ordonner deux situations de non-unanimité, soit A et B. Un tenant de l'unanimité et de l'agnosticisme devrait rester indifférent au fait que les préférences dominantes soient celles des maîtres ou celles des esclaves. En ce sens, la condition d'unanimité peut bien nous interdire de décréter que la situation $B$ est supérieure à la situation C ( $c^{\prime}$ est l'inverse qui est vrai), cependant elle ne permet aucunement d'établir la supériorité de A sur B. En d'autres termes, le raisonnement de Buchanan ne peut établir la supériorité du statu quo : si la situation d'unanimité est par définition la meilleure, aucun critère ne nous permet de trancher entre des situations imparfaites.

En fait, loin de découler du critère d'unanimité, la préférence de Buchanan pour le statu quo tient plutôt à son estimation des conséquences possibles d'un changement unilatéral : un tel

65 Voir Jules Coleman, «Some Well and Still Other III Founded Skepticism About Constitutionalism», in MacKenzie, Richard, éd., Constitutional Economics : Constraining the Economic Powers of Government, Lexington, Mass., Lexington Books, 1984, p. 144-155. 
changement produirait effectivement chez la partie se jugeant lésée un sentiment $d$ 'iniquité qui risquerait "de miner sévèrement le sentiment de légitimité sans lequel les gouvernements modernes ne pourraient pas survivre ${ }^{66}$ "? Or, un affaiblissement de la légitimité de l'État nous rapproche du désordre absolu, lequel est pire que tout, en raison de l'incertitude qu'il génère. Certes, bien des situations qui, à certains égards, peuvent sembler peu enviables demeurent malgré tout préférables à la guerre de tous contre tous. Mais est-on nécessairement condamné à l'alternative de la jungle hobbesienne et du statu quo ? Existe-t-il des états intermédiaires qui ne se confondent pas avec l'anarchie complète ? En fait, la position de Buchanan ne tient que si le pire est toujours certain, mais de cela aussi, l'agnostique devrait douter. On peut, par exemple, considérer qu'un peu de désordre, qu'une perte limitée et temporaire de la légitimité gouvernementale sont le prix à payer pour le redressement d'une injustice et que le versement d'une compensation à ceux qui ont profité de cette injustice serait immoral. On peut même en certains cas estimer qu'un état de choses est tellement inique qu'un détour par l'anarchie se justifie $^{67}$. Certes, pour l'agnostique, on commet alors la faute d'universaliser une préférence subjective, mais celui qui invoque en dernier ressort la nécessité de sauvegarder la légitimité du gouvernement fait-il autre chose ? La "paix sociale» est certes une valeur estimable, mais, aux yeux de l'agnostique intransigeant, en quoi l'est-elle plus qu'une conception de la justice qui amènerait à condamner l'octroi de tarifs protectionnistes à certaines industries, la reconnaissance de privilèges à une catégorie particulière de citoyens ou le maintien d'une partie de la population dans l'esclavage?

Buchanan n'est donc pas fondé à prétendre que «le rôle dominant de la liberté individuelle est imposé par la reconnaissance de la méthodologie individualiste et non par (des) évaluations subjectives $^{68}$ ". En passant de la reconnaissance du fait de la diversité des préférences individuelles à l'idée d'une inviolabilité de l'autonomie axiologique, il quitte le terrain strictement méthodologique. En choisissant de définir la liberté par le respect absolu des préférences individuelles, Buchanan adopte une position

\footnotetext{
66 «Dismantling the Welfare Staten, p. 184.

67 On peut voir dans la guerre civile américaine un cas de ce genre.

The Limits of Liberty, p. 2.
} 
clairement axiologique, puisqu'il accorde alors plus de poids aux préférences présentes qu'aux préférences passées et juge plus intolérables les changements unilatéraux auxquels l'on voudrait procéder aujourd'hui que des situations pourtant illégitimes à l'aune du diagnostic contractualiste. Dire qu'on ne peut fonder en science la suprématie d'une valeur est une chose. En déduire que toutes les normes se valent et de là que le statu quo est préférable à toute situation où le jugement des uns s'imposerait à celui des autres est fort différent. Voilà un exemple très net d'effet idéologique : si vous acquiescez à l'individualisme méthodologique, nous dit Buchanan, vous devrez alors admettre ma conception de la liberté individuelle et la déontologie qui l'accompagne. Mais en aucune façon l'accord avec les analyses positives de Buchanan n'implique que l'on renonce à tenir certaines valeurs pour fondamentalement justes. On peut fort bien tout à la fois refuser de voir dans l'activité politique la recherche d'une vérité et tenir que certains droits individuels constituent des contraintes absolues $^{69}$. On peut admettre la pluralité des préférences individuelles et exclure de la discussion celles qui feraient obstacle à la conclusion du contrat. Enfin, celui qui cherche à comprendre pourquoi des contractants rationnels peuvent choisir de se soumettre à la coercition sera fort avisé de prendre pour point de départ l'idée d'unanimité; rien ne l'autorise pour autant à conclure que I'unanimité doit être érigée en critère suprême.

\section{Conclusion : savoir et se délivrer du choix}

II nous paraît opportun, en terminant, de replacer le projet intellectuel de Buchanan dans son contexte idéologique. S'il appartient à la même famille que Hayek ou Friedman, Buchanan n'explique pas de la même façon qu'eux l'extension de la gestion gouvernementale de la vie économique et sociale. Alors que les premiers y voient le triomphe de l'hubris, la victoire d'un rationalisme devenu irrationnel pour avoir outrepassé ses limites, Buchanan $y$ lit le résultat involontaire de l'agrégation des préférences et des actions d'individus banalement rationnels. Mais la sérénité de cette analyse débouche paradoxalement sur un pessimisme marqué quant aux possibilités de revenir par la

os Tel nous apparaît, par exemple, le libéralisme défendu par le philosophe John Rawls ou le juriste Ronald Dworkin. Voir, du premier, A Theory of Justice, Cambridge, Harvard University Press, 1971 et, du second, Taking Rights Serious/y, Cambridge, Harvard University Press, 1977. 
persuasion à l'État limité. Si Léviathan, c'est nous, la propagation de l'évangile libéral ne sera pas d'un grand secours. Pis encore, le risque est grand qu'un gouvernement acquis aux idées libérales impose avec autorité des réformes impopulaires qui entraîneront une réaction vive et un rejet du libéralisme. Par conséquent, Buchanan privilégie le démantèlement progressif de l'État-providence. Pour minimiser les réticences et les oppositions, il est préférable de faire porter la discussion sur la forme plutôt que sur la substance, sur les règles de prise de décision plutôt que sur le contenu des décisions, étant bien sûr entendu que le processus n'est pas sans effet sur le résultat (n'est-ce pas de cette hypothèse qu'est issue la théorie du public choice ?). En comparaison des thérapies de choc proposées par Hayek (dénationalisation rapide de la monnaie) et Friedman (remplacement de l'ensemble des mesures sociales par un système d'impôt négatif), la "réforme pragmatique» mise de l'avant par Buchanan relève de la médecine douce ${ }^{70}$. Mais en présentant sa déontologie comme une conséquence nécessaire de l'individualisme méthodologique, Buchanan participe en même temps au projet de fonder le libéralisme uniquement sur la science. Von Mises n'est-il pas simplement plus cru lorsqu'il décrit le libéralisme comme "une idéologie scientifique (...) dérivée de ces pures sciences que sont l'économie et la sociologie" et accorde à ces dernières le mérite d'avoir établi de façon irréfutable la supériorité du capitalisme sur "tous les autres systèmes d'organisation sociale concevables ${ }^{71} "$ ? Friedman, pour sa part, nourrit-il une autre ambition lorsqu'il déclare caducs les conflits de valeurs et estime que "les divergences en matière de politique économique (...) peuvent en principe être éliminées par les progrès de l'économie positive ${ }^{72}$ "? Certes, on dira qu'un Hayek demeure profondément sceptique devant ce rationalisme utilitariste. II n'en croit pas moins que la décision d'intervenir ou non dans le fonctionnement de l'ordre spontané pour corriger des conséquences que l'on déplore est une question purement

70 Voir Hayek, Friedrich, Denationalisation of Money. The Argument Refined, Londres, Institute of Economic Affairs, 1978 et Friedman, Milton et Rose Friedman, Free to Choose. A Personal Statement, New York, Harcourt Brace Jovanovich, 1979.

7 von Mises, Ludwig, Liberalism in the Classical Tradition, Irvington-on-Hudson, Foundation for Economic Education, 1985 [1927], p. 88.

12 Friedman, Milton, "The Methodology of Positive Economics", in Essays in Positive Economics, Chicago, University of Chicago Press, 1953, p. 5. 
factuelle, qui relève à ce titre du seul tribunal de la science. Buchanan ne conteste pas l'inéluctabilité des conflits entre les fins, mais c'est pour affirmer du même souffle qu'en dépit de cela, un accord sur les moyens est non seulement possible, mais suffisant pour faire advenir une société libre et pacifiée. Bien sûr, aucun de ces auteurs ne remet formellement en cause la distinction entre l'être et le devoir-être et aucun n'écrirait noir sur blanc qu'une position méthodologique ou une analyse scientifique peuvent commander l'adoption de valeurs données. Mais si la primauté de la liberté individuelle, si l'ordre économique et social, les règles de justice ou le contenu du contrat social peuvent être établis malgré les conflits entre les valeurs, sur quoi ceux-ci portent-ils donc? Pour Buchanan, comme pour tous ceux qui prétendent fonder scientifiquement leur libéralisme, les dilemmes axiologiques peuvent toujours recevoir une solution procédurale : il ne s'agit pas tant ici d'une confusion entre les faits et les valeurs que d'un refus de laisser à celles-ci autre chose qu'une place congrue dans les affaires humaines, d'une volonté de les ravaler en quelque sorte au même rang que les préférences en matière d'esthétique. La règle d'or devient : des fins et des valeurs tu ne discuteras point; à faire ton bonheur moyens et règles suffiront. Or, si la science nous aide à comprendre des fragments du réel, elle ne peut en aucune façon nous dicter notre conduite. De la même façon, en nous enjoignant de retracer les microfondements des phénomènes sociaux, en insistant sur le caractère non délibéré de ceux-ci, en nous incitant à nous défier des concepts collectifs, l'individualisme méthodologique contribue à la résolution d'énigmes particulières. Mais il ne peut pas nous délivrer du choix éthique ni dissiper l'incertitude qui s'attache à cette sphère de l'existence humaine. Comme l'écrivait récemment un auteur libéral qui ne partage pas cette illusion : «Nous sommes condamnés à choisir, et chaque choix peut entraîner une perte irréparable ${ }^{73}$ ". Un monde tel que le voient les néo-libéraux, c'est-à-dire un monde où l'efficacité et la moralité se réconcilieraient toujours aisément, serait sans doute désirable. Mais ce ne serait pas notre monde.

73 Berlin, Isaiah, "On the Pursuit of the Ideal», in The New York Review of Books, 1988, 17 mars, p. 15. 